\title{
CRITERIOS DE LA TEORÍA DE PROBABILIDADES EN LA EVALUACIÓN DE CONTINGENCIAS EN EMPRESAS MINERAS PERUANAS
}

\author{
CRITERIA OF PROBABILITIES THEORY IN CONTINGENCIES ASSESSMENT IN THE \\ PERUVIAN MINING COMPANIES
}

Rosa Milagros Castañeda Moreano

Pontificia Universidad Católica del Perú

Lima, Perú

ORCID: https://orcid.org/0000-0003-3097-8504

Correo electrónico: rcastanedamoreano@gmail.com

\section{RESUMEN}

Objetivo: Comprender las definiciones y propiedades de la Teoría de Probabilidades a fin de considerarlas en la evaluación de la información financiera sobre contingencias, enfatizando el concepto de probabilidad. Método: La investigación es de enfoque cualitativo, de tipo exploratorio y parcialmente descriptivo, de diseño no experimental y transversal, con supuestos hipotéticos, sobre la base del análisis de información, estudio de casos y entrevistas semiestructuradas. Resultados: Las contingencias son reveladas en las notas más que ser reconocidas en los estados financieros. El nivel de revelación no es suficiente en cuanto a los juicios aplicados en las estimaciones, así como en la impracticabilidad de los mismos y su racionalidad. Conclusiones: El concepto de probabilidad en las Normas Internacionales de Información Financiera difiere del concepto establecido en la Teoría de Probabilidades. La estimación de las probabilidades debe considerar la naturaleza y características de la contingencia y la probabilidad de ocurrencia del evento, traducido en ganar o perder un litigio, considerando el enfoque bayesiano, puesto que mide la creencia sobre la ocurrencia de un evento.

Palabras clave: Contingencias; estimación; evento; incertidumbre; probabilidad.

\begin{abstract}
Objective: Comprehend the definitions and properties of the Probability Theory in order to consider in the evaluation of financial information on contingencies, emphasizing the probability concept. Method: The research is a qualitative approach, of exploratory type and partially descriptive, of non-experimental and cross-sectional design, with hypothetical assumptions, based on the information analysis, case studies and semi structured interviews. Results: Contingencies are revealed in notes rather than being recognized in the financial statements. The level of revelation is not enough in terms of judgments applied in the estimates, as well as in their impracticability and rationality. Conclusions: The concept of probability in international financial reporting standards differs from the concept stablished in the Probabilities Theory. The probabilities estimation should consider the nature and characteristics of the contingency and the probability of the event occurrence, translated into winning or losing a dispute, considering the Bayesian approach, since it measures the belief about the occurrence of an event.
\end{abstract}

Keywords: Contingencies; estimation; event; uncertainty; probability. 


\section{INTRODUCCIÓN}

Desde el año 2011, las empresas mineras peruanas reportan información financiera sobre la base de las Normas Internacionales de Información Financiera (en adelante, NIIF), la cual es pública. Ante situaciones en las cuales las empresas no tienen el control de los factores externos, como por ejemplo: la presencia de incertidumbre, la falta de un criterio que permita la objetividad de los procesos de decisión, aunado a la ausencia de una norma contable que excluye el uso de cálculo de probabilidades con bases matemáticas por parte de los gestores y asesores de dichas empresas; estos, se constituyen es aspectos relevantes para la toma de decisiones, puesto que implica la aplicación de conocimiento, experiencia y criterio profesional. La probabilidad es un concepto que requiere una interpretación adecuada, para ello la probabilidad bayesiana permite la medición del grado de creencia sobre la ocurrencia de un evento.

La investigación analiza las condiciones dadas por los litigios que enfrentan las empresas mineras peruanas y su efecto en sus estados financieros, a partir del cual se ha identificado un criterio subjetivo en cuanto a la estimación de probabilidades para el tratamiento contable de las contingencias originadas por litigios, lo cual podría llevar a una equívoca interpretación de la información provista por los estados financieros y consecuentemente, afectaría la toma de decisiones sobre la situación financiera y los resultados de dichas empresas. Asimismo, analiza la discrepancia que existe sobre la interpretación de la probabilidad, a nivel de la NIC 37 - Provisiones, Pasivos Contingentes y Activos Contingentes (en adelante, NIC 37) y la Teoría de Probabilidades, la cual conlleva a una comprensión de los aspectos matemático y filosófico de la probabilidad, a fin de entender la problemática en su totalidad e identificar los criterios adecuados para la evaluación de contingencias, así como los criterios de reconocimiento de probabilidad.

En el desarrollo de la investigación, no se han identificado trabajos de investigación que hayan abordado la misma situación problemática del presente trabajo. Se tienen las referencias que se desarrollan en líneas siguientes:

El sistema axiomático de la Teoría de Probabilidades fue estructurado por Andréi Kolmogórov en 1933. En tal sentido, definió los siguientes componentes:

- E es un experimento aleatorio,

- $\Omega$ es el espacio muestral asociado,

- A es un evento cualquiera de $\Omega$ (este último, el espacio muestral)
Sobre los cuales, desarrolló los siguientes axiomas:

1. $\mathrm{P}(\mathrm{A}) \geq 0$, se interpreta como: La probabilidad de cualquier evento A es mayor o igual que cero. Es decir, no es negativo.

2. $P(\Omega)=1$, se interpreta como: La probabilidad de que ocurra cualquier evento del espacio muestral es igual a 1 .

3. Si A y B son eventos definidos sobre el espacio muestral $\Omega$, tales que $A \cap B=\varnothing$, entonces, $P$ (A $\mathrm{U} B)=\mathrm{P}(\mathrm{A})+\mathrm{P}(\mathrm{B})$, se interpreta como: La probabilidad de que ocurra el evento A o el evento $B$, es igual a la probabilidad de que ocurra el evento A más la probabilidad de que ocurra B, sí y solo sí la intersección de dichos es nula; es decir, son disjuntos.

Thomas Bayes estudió el problema de la determinación de la probabilidad de las causas de un evento, a través de los efectos observados de otros eventos. Su teorema consiste en lo siguiente: Sea el evento A, el cual forma una partición del espacio muestral $\Omega$; luego, para cualquier otro evento (no nulo) B de $\Omega$, se tiene que:

$P(A \mid B)=P(A) \cdot \frac{P(B \mid A)}{P(B)} \quad($ García, 2015, p.124)

De los estudios realizados por Bayes surge la probabilidad bayesiana, en contraposición a la probabilidad objetiva (debido a que esta última mide la probabilidad "real" y "objetiva" y se determina "a priori”). La probabilidad bayesiana tiene como fin la medición del grado de creencia sobre la ocurrencia de un evento.

McGrayne (2012) efectuó el análisis del Teorema de Bayes considerando el contexto histórico y científico anterior a él a partir del siglo XVI, hasta los impactos generados por su Teorema a inicios del siglo XXI. Sus principales conclusiones son: "[...] la regla de Bayes [...] se hallaba vinculada a una interrogante [...]: la de cómo analizar las pruebas, cómo modificar nuestro punto de vista conforme vamos adquiriendo nueva información, y cómo tomar decisiones racionales frente a la incertidumbre" (p. 11). En ese sentido, la nueva información obtenida para el análisis de un evento puede tener un impacto importante en la toma de decisiones, sobre todo en caso de eventos que contienen incertidumbre, como por el ejemplo las contingencias. "El teorema de Bayes permite valorar una creencia, [...] no sólo es posible adquirir conocimiento, aunque nos falten datos o éstos resulten inadecuados, sino que [...] el saber puede obtenerse partiendo de aproximaciones e incluso de la ignorancia” (p. 12). 
Por otro lado, Bernardo y Smith (2000) afirman:

[...] las probabilidades son siempre grados personales de creencia, en el sentido de que son una representación numérica de la relación de incertidumbre personal de quien toma las decisiones entre eventos. Además, las probabilidades siempre están condicionadas a la información actualmente disponible. (p.35)

Por lo expuesto, algunos investigadores presuponen que la asignación de probabilidad es objetiva, es decir, independiente del sujeto que la calcula. Sin embargo, tomando en consideración la probabilidad bayesiana expuesta, y analizando los criterios que provienen de ella, el proceso de evaluación de contingencias depende del marco de información disponible a la fecha de evaluación, debido a las siguientes razones:

i. La Gerencia de una empresa junto con la participación de sus asesores legales, por lo general, asignarían probabilidades en función al conocimiento especializado obtenido a través de la experiencia y otros casos similares; $y$

ii. En ningún caso se apoyarían en estadísticas obtenidas de eventos pasados similares, con la rigurosidad matemática que se requiere.

La investigación considera los aspectos relevantes del Marco Conceptual para la Información Financiera (2018), así como la NIC 37 y la NIC 1 - Presentación de Estados Financieros (en adelante NIC 1), cubre los años terminados el 31 de diciembre de 2015 al 2018 y toma en cuenta la información de estados financieros auditados de las empresas mineras que reportan a la Superintendencia de Mercado de Valores (en adelante SMV).

Por otro lado, los supuestos acerca de la incertidumbre en la estimación, refieren a las estimaciones que involucren una mayor dificultad, subjetividad o complejidad en el juicio profesional. Ante un mayor número de supuestos o variables consideradas en el análisis, existe la posibilidad de un juicio profesional más subjetivo y complejo, que generen cambios en los estados financieros. Sin embargo, en relación a la impracticabilidad, en cuanto a la estimación de los posibles efectos de la incertidumbre, la NIC 1 dice:

Algunas veces es impracticable revelar el alcance de los posibles efectos de una hipótesis u otra fuente de incertidumbre en la estimación al final del periodo del que se informa. En tales casos, la entidad revelará que es razonablemente posible, sobre la base del conocimiento existente, que los desenlaces producidos dentro del siguiente periodo contable que sean diferentes de los supuestos utilizados, podrían requerir ajustes significativos en el importe en libros del activo o pasivo afectado. En cualquier caso, la entidad revelará la naturaleza y el importe en libros del activo o pasivo del específico (o de la clase de activos o pasivos) afectado por el supuesto en cuestión. (International Accounting Standards Board, 2015, párr. 131)

A nivel de racionalidad, Mosterín (2008) desarrolla el concepto de creencia racional como aquel más operativo y manejable, el cual ocupa una posición intermedia entre el opinar y el saber. Por otro lado, afirma que una creencia racional está justificada en función de la base informativa disponible en un momento determinado. De acuerdo con este autor, la definición de creencia racional, requiere dos condiciones: la afirmación de la creencia y la justificación de la misma: idea analítica; comprobada directamente; tal creencia se "constituye en una opinión generalmente compartida por la comunidad científica pertinente" (p. 24); presenta testimonios fiables, directos e independientes; $y$ no presenta ideas contradictorias.

En ese contexto, la investigación busca responder la siguiente interrogante: ¿Cómo se deberían describir los criterios de análisis de probabilidad en la evaluación de las contingencias y su clasificación establecida en la NIC 37, para las empresas mineras peruanas? A partir de ello, el objetivo fue describir los criterios de análisis de probabilidad en la evaluación de las contingencias y el análisis de suficiencia de la clasificación establecida por la NIC 37 para las empresas mineras peruanas.

La investigación se justifica en la observación de los criterios que aplican las empresas mineras peruanas respecto de las contingencias, en cuanto al análisis de probabilidad. Si bien la NIC 37 define las condiciones que la Gerencia debería adoptar al momento de reconocer una provisión por contingencias (además de ser un aspecto que requiere el juicio profesional, criterio, experiencia y conocimiento de la industria), aún se puede observar que hay diferencias en las revelaciones y estimaciones presentadas en los estados financieros de dichas empresas. Asimismo, el análisis de probabilidad ante situaciones de incertidumbre, podría resultar en provisiones, pasivos contingentes o simplemente no ser presentados en los estados financieros.

La principal limitación de la investigación fue la falta de disponibilidad de datos estadísticos sobre las contingencias y la falta de revelación específica de los supuestos asumidos en el juicio profesional para la emisión de estados financieros.

\section{MATERIALES Y MÉTODOS}

La investigación es de enfoque cualitativo, de tipo exploratorio y parcialmente descriptivo, de diseño no experimental 
y transversal, con el uso del método deductivo-inductivo; por ello, se han enunciado supuestos hipotéticos, que se afinaron a lo largo de la investigación. En ese sentido, la investigación se centró en presentar aspectos relevantes respecto de la probabilidad en la evaluación de contingencias a las que están expuestas las empresas mineras peruanas, así como la comprensión de los conceptos matemáticos de la Teoría de Probabilidades en la evaluación de probabilidad en términos de grado de creencia sobre la ocurrencia del desenlace sobre un litigio, ya sea a favor o en contra de la empresa.

Para ello, se efectuó la recolección de información relevante de los estados financieros auditados de las empresas mineras peruanas, así como el estudio de casos múltiple de tres tipos de contingencia: ambientales, laborales y tributarias y su contraste con los requerimientos de las NIIF y las definiciones y propiedades de la Teoría de Probabilidades, así como entrevistas semiestructuradas, realizadas tanto a profesionales contables que se desempeñan como revisores de estados financieros o como auditores de estados financieros, cuya selección se centró en su desempeño, experiencia y conocimiento del sector minero. La unidad de estudio fueron las contingencias reportadas en sus estados financieros auditados y publicados por la SMV.

La selección de casos se efectuó en función a la significatividad de los mismos en cuanto a sus características y la suficiencia de la información disponible (naturaleza, reconocimiento y revelación). Para el sector minero, cada tipo de contingencia toma importancia debido a las características propias de sector, a la regulación y al impacto que puede generar. Los casos seleccionados, en función a la clasificación de contingencias, se presentan a continuación:

1. Contingencias Ambientales: Sociedad Minera El Brocal S.A.A.

2. Contingencias Laborales: Compañía Minera San Ignacio de Morococha S.A.A.
3. Contingencias Tributarias: Sociedad Minera Corona S.A.

\section{RESULTADOS}

A partir de la información de los estados financieros auditados de las empresas mineras peruanas, se identificó que la proporción de las contingencias provisionadas es menor al de las contingencias reveladas, ambas en relación a la posición del pasivo total al cierre anual de los años analizados, lo cual indica que las contingencias, en su mayoría, son reveladas en las notas más que ser reconocidas en los estados financieros. El estudio de casos, presenta lo siguiente:

La tabla 1 presenta la evolución de las proporciones de las contingencias provisionadas entre el pasivo total. En el caso de Sociedad Minera El Brocal S.A.A. el saldo de provisiones por contingencias fue incrementándose progresivamente de US\$0,5 millones en 2015 a US\$6 millones en 2018; en Compañía Minera San Ignacio de Morococha S.A.A. pasó de $\mathrm{S} / 3,8$ millones en 2015 a un promedio de S/6,3 millones de 2016 a 2018; mientras que, Sociedad Minera Corona S.A., pasó de US\$0,4 millones en 2015 a US $\$ 2,7$ millones en 2018. En las tres empresas analizadas se observó un incremento en las contingencias provisionadas, lo cual muestra un reconocimiento progresivo de las contingencias a medida que se resuelven o cuando se obtienen mayores indicios sobre el posible desenlace de dichas contingencias.

La Tabla 2 presenta el comparativo de las proporciones de las contingencias reveladas entre el pasivo total. En el caso de Sociedad Minera El Brocal S.A.A., las contingencias reveladas ascendieron a US\$9,6 millones en 2015 y se mantuvo alrededor de US\$4 millones de 2016 a 2018. En el caso de Sociedad Minera Corona S.A. el promedio de contingencias reveladas se mantuvo en alrededor de US\$230 millones en los cuatro años. A diferencia de la tabla anterior, el comportamiento se muestra relativamente estable entre los años 2015 al 2018, salvo en el caso de

Tabla 1

Proporción de las contingencias provisionadas entre el pasivo total por los años 2018, 2017, 2016 y 2015

\begin{tabular}{lcccc}
\hline Empresa/Año & $\mathbf{2 0 1 8}$ v.a. & $\mathbf{2 0 1 7}$ v.a. & $\mathbf{2 0 1 6}$ v.a. & $\mathbf{2 0 1 5}$ v.a. \\
\hline Sociedad Minera El Brocal S.A.A. & 0,02 & $<0,01$ & $<0,01$ & $<0,01$ \\
Compañía Minera San Ignacio de Morococha S.A.A. & 0,04 & 0,04 & 0,03 & 0,02 \\
Sociedad Minera Corona S.A. & 0,06 & 0,05 & 0,05 & 0,01 \\
\hline
\end{tabular}

Nota: v.a. = expresado en valores absolutos. Adaptado de Sociedad Minera El Brocal S.A.A. (2018), Compañía Minera San Ignacio de Morococha S.A.A. (2018) y Sociedad Minera Corona S.A. (2018)

Fuente: Elaboración propia 
Tabla 2

Proporción de las contingencias reveladas entre el pasivo total por los años 2018, 2017, 2016 y 2015

\begin{tabular}{lcccc}
\hline Empresa/Año & $\mathbf{2 0 1 8}$ v.a. & $\mathbf{2 0 1 7}$ v.a. & $\mathbf{2 0 1 6}$ v.a. & $\mathbf{2 0 1 5}$ v.a. \\
\hline Sociedad Minera El Brocal S.A.A. & 0.01 & 0.01 & 0.01 & 0.03 \\
Compañía Minera San Ignacio de Morococha S.A.A. & $<0.01$ & $(1)$ & $(1)$ & $(1)$ \\
Sociedad Minera Corona S.A. & 4,67 & 4,93 & 3,62 & 4,18 \\
\hline
\end{tabular}

Nota: v.a. = expresado en valores absolutos; $(1)$ = Contingencias reveladas en notas, pero no estimadas por la empresa.

Fuente: Elaboración propia

Compañía Minera San Ignacio de Morococha S.A.A. pues no reveló del año 2015 al 2017 los importes estimados de las contingencias y solo indicó que la Gerencia y sus legales mantienen una expectativa mediana de resultado favorable (Compañía Minera San Ignacio de Morococha S.A.A., 2018, pp. 60-64).

Como resultado del análisis de estados financieros auditados, se observó que las empresas mineras analizadas cumplen parcialmente los requerimientos establecidos en el párrafo 122 de la NIC 1 (revelación de juicios en la aplicación de políticas contables y juicios que involucren estimaciones), y presentaron, en las notas, las siguientes revelaciones:

(i) Respecto de los juicios en la aplicación de las políticas contables: moneda funcional y préstamos a subsidiaria (Compañía Minera San Ignacio de Morococha S.A.A., 2018, pp. 13-14), contingencias y fecha de inicio de la etapa de producción (Sociedad Minera El Brocal S.A.A., 2018, pp. 2324). Sociedad Minera Corona S.A. (2018) efectuó una revelación general (p. 22).

(ii) Respecto de los estimados y supuestos contables críticos: provisión para procesos administrativos y laborales (Compañía Minera San Ignacio de Morococha S.A.A., 2018, pp. 12-13), contingencias (Sociedad Minera Corona SA., 2018, pp. 23-25).

En relación a los párrafos 125, 129 y 131 de la NIC 1, respecto a las causas de incertidumbre en las estimaciones, las empresas mineras sujetas a análisis revelaron lo siguiente:

[...] supuestos claves relacionados con el futuro $y$ otras fuentes clave de estimaciones de incertidumbres a la fecha de los estados financieros, y que conllevan un alto riesgo de ocasionar ajustes significativos sobre los importes en libros de los activos y pasivos durante el próximo periodo. La Compañía ha basado sus estimados y supuestos contables tomando en consideración aquellos parámetros disponibles al momento de la preparación de los [...] estados financieros. Sin embargo, las circunstancias y los supuestos actuales sobre los acontecimientos futuros podrían variar debido a cambios en el mercado y a circunstancias nuevas que pudieran surgir más allá del control de la Compañía. Los cambios son reflejados en los supuestos al momento de ocurrir. (Sociedad Minera El Brocal S.A.A., 2018, p. 24)

La preparación de los estados financieros [...] de acuerdo con las NIIF requiere que la Gerencia de la Compañía realice juicios, estimaciones y supuestos que afectan la aplicación de las políticas contables y los montos de los activos, pasivos, ingresos y gastos informados. Los resultados reales pueden diferir de estas estimaciones. [...] Las estimaciones y supuestos relevantes son revisados regularmente. Las revisiones de las estimaciones contables son reconocidas en el periodo en que la estimación es revisada y en cualquier periodo futuro afectado. (Compañía Minera San Ignacio de Morococha S.A.A., 2018, pp. 11-12)

Los estimados y criterios usados son continuamente evaluados y se basan en la experiencia histórica y otros factores, incluyendo la expectativa de ocurrencia de eventos futuros que se consideran razonables de acuerdo con las circunstancias. [...] La Compañía efectúa estimaciones y supuestos respecto del futuro. Las estimaciones contables resultantes, por definición, muy pocas veces serán iguales a los resultados reales. En opinión de la Gerencia, estas estimaciones se efectuaron sobre la base de su mejor conocimiento de los hechos relevantes y circunstancias a la fecha de preparación de los estados financieros; sin embargo, los resultados finales podrán diferir de las estimaciones incluidas en los estados financieros. La Gerencia de la Compañía no espera que las variaciones, si las hubiera, tengan un efecto importante sobre los estados financieros. (Sociedad Minera Corona S.A., 2018, pp. 22-23)

Se identificaron dos aspectos que se cumplieron parcialmente en los casos analizados: (i) no se explicaron las causas de la impracticabilidad (puesto que la NIC 1 solo hace referencia a efectuar todos los esfuerzos posibles) de 
revelar los posibles efectos de una hipótesis u otra fuente de incertidumbre en la estimación; y (ii) no se detalló la información presente o no se justificaron las condiciones (en cuanto a su característica de "razonablemente posible") que originarían los posibles desenlaces en el siguiente periodo bajo supuestos diferentes a los asumidos.

Considerando lo expuesto por Mosterín (2008) en relación a la creencia racional, se observó en los estados financieros de las empresas mineras analizadas, que solo cumplen con afirmar una creencia, es decir, dar cuenta o expresar una opinión o una afirmación sobre una conclusión (los supuestos realizados acerca del futuro, las causas de incertidumbre en la estimación, y según sea el caso, aquellos que tengan un riesgo significativo de ocasionar ajustes a los estados financieros, a nivel de su naturaleza e importe); mientras que no se observó en los estados financieros la justificación detallada de dicha creencia. En consecuencia, dicha afirmación (o creencia) carecería de racionalidad en ese sentido.

Como resultado del análisis de estados financieros auditados, se observó que las empresas mineras analizadas no efectuaron una revelación sobre los criterios de análisis de la probabilidad en la evaluación de las contingencias. Como resultado de las entrevistas semiestructuradas (ver tabla 3), se identificó que el criterio aplicado en el análisis de la probabilidad es subjetivo a partir de un enfoque de análisis cualitativo, además de la opinión de los asesores legales en función a su experiencia y a la jurisprudencia existente.
Respecto al análisis de suficiencia de la clasificación establecida por la Guía de Implementación de la NIC 37, como resultado de las entrevistas semiestructuradas realizadas, las empresas mineras realizaron una evaluación bajo un enfoque cualitativo según los siguientes niveles: probable (probabilidad mayor a 50\%), posible (probabilidad de 50\%) y remota (probabilidad menor a 50\%). En un caso, se amplió a cinco niveles: inminente (probabilidad mayor a $80 \%$ ), altamente probable (probabilidad mayor entre $60 \%$ y $80 \%$ ), probable (probabilidad entre $35 \%$ y $60 \%$ ), poco probable (probabilidad entre 15\% y $35 \%$ ) y remoto (probabilidad menor a $15 \%$ ), lo cual si bien contribuye a segmentar los niveles de probabilidad de manera más extensiva, se basa en el criterio subjetivo de la Gerencia y en la opinión de los asesores legales.

La Teoría de Probabilidades aportó, en cuanto a los criterios de análisis de la probabilidad en la evaluación de las contingencias, lo siguiente:

- El análisis de la probabilidad de los fenómenos involucra aleatoriedad: (i) pueden tener más de un posible resultado el cual no se puede predecir con seguridad, (ii) se puede describir el conjunto de todos los resultados posibles y (iii) puede repetirse muchas veces. Para el análisis de contingencias, estas pueden tener dos resultados: que se gane o se pierda un litigio, es posible describir todos los resultados posibles que tuviese el litigio a nivel de las instancias pertinentes y se puede analizar periódicamente.

Tabla 3

Resumen de las entrevistas realizadas a profesionales contables que se desempeñan como revisores y auditores de estados financieros

\begin{tabular}{|c|c|c|c|c|c|}
\hline $\begin{array}{l}\text { Temas/ } \\
\text { Entrevistado }\end{array}$ & $\begin{array}{l}\text { Jefe de Reporte } \\
\text { Financiero de una } \\
\text { empresa minera }\end{array}$ & $\begin{array}{l}\text { Especialista en } \\
\text { Análisis Financieros } \\
\text { de Gerencia de una } \\
\text { empresa minera }\end{array}$ & $\begin{array}{l}\text { Profesional contable } \\
\text { con especialidad en } \\
\text { aspectos legales y } \\
\text { tributarios }\end{array}$ & $\begin{array}{l}\text { Socio Líder en } \\
\text { Minería de una } \\
\text { Sociedad de } \\
\text { Auditoría }\end{array}$ & $\begin{array}{l}\text { Gerente Senior de } \\
\text { Auditoria Financiera } \\
\text { en el Sector Minero } \\
\text { de una Sociedad de } \\
\text { Auditoria }\end{array}$ \\
\hline $\begin{array}{l}\text { Niveles de } \\
\text { probabilidad } \\
\text { considerados }\end{array}$ & $\begin{array}{l}\text { Inminente } \\
\text { Altamente probable } \\
\text { Probable } \\
\text { Poco probable } \\
\text { Remoto }\end{array}$ & $\begin{array}{l}\text { Probable } \\
\text { Posible } \\
\text { Remoto }\end{array}$ & $\begin{array}{l}\text { Probable } \\
\text { Posible } \\
\text { Remoto }\end{array}$ & $\begin{array}{l}\text { Probable } \\
\text { Posible } \\
\text { Remoto }\end{array}$ & $\begin{array}{l}\text { Probable } \\
\text { Posible } \\
\text { Remoto }\end{array}$ \\
\hline $\begin{array}{l}\text { Aplicación de } \\
\text { criterio } \\
\text { profesional }\end{array}$ & $\begin{array}{l}\text { Basado en la expe- } \\
\text { riencia profesional y } \\
\text { conocimiento de las } \\
\text { normas }\end{array}$ & $\begin{array}{l}\text { Basado en la expe- } \\
\text { riencia profesional y } \\
\text { conocimiento de las } \\
\text { normas }\end{array}$ & $\begin{array}{l}\text { Criterio subjetivo } \\
\text { podría generar un } \\
\text { resultado contrario al } \\
\text { esperado }\end{array}$ & $\begin{array}{l}\text { Basado en el juicio, } \\
\text { conocimiento de las } \\
\text { normas y experien- } \\
\text { cias similares en el } \\
\text { sector, apoyando en } \\
\text { los asesores legales }\end{array}$ & $\begin{array}{l}\text { Basado en el criterio, } \\
\text { conocimiento del } \\
\text { sector. } \\
\text { Se considera el cri- } \\
\text { terio de los asesores } \\
\text { legales }\end{array}$ \\
\hline
\end{tabular}

Fuente: Elaboración propia. 
- Una contingencia se analiza atendiendo su tipología (ambiental, laboral o tributaria), a través de su comportamiento en el pasado; es decir, los resultados obtenidos en las instancias a lo largo del tiempo de duración del litigio, así como de la jurisprudencia disponible.

La probabilidad de ocurrencia de un evento se expresa numéricamente entre los rangos 0 (incertidumbre) y 1 (certeza). Una data suficiente permite el análisis de hechos pasados y sus resultados.

- El uso de la probabilidad bayesiana permite la medición del grado de creencia sobre la ocurrencia de un evento. Se entiende que este tipo de creencia tiene un nivel de racionalidad, según lo expuesto por Mosterín (2008), en el sentido de que, para establecer la probabilidad de que la Compañía A gane un litigio, es necesario medir la probabilidad de que tal evento ocurra, a partir de los cuales se obtienen unos resultados y su correspondiente interpretación, lo cual se convertiría en la teoría que sustenta el desenlace a favor de la Compañía A. Es así que se tienen los siguientes elementos de análisis: (i) la probabilidad a priori o inicial (proveniente de las estadísticas de los resultados obtenidos en el pasado, esto es del conocimiento previo que se tenga), y (ii) la actualización de la información disponible (que corresponde a nueva información referente al estado de la contingencia).

- La probabilidad bayesiana, permite contar con un resultado con mayor validez y carga de información, demostrando que se trata de una creencia racional, puesto que permite comparar las probabilidades obtenidas después de la ocurrencia de un evento de interés, y se convierte en una herramienta útil para la toma de decisiones.

\section{DISCUSIÓN}

Si bien la NIC 1 establece la revelación de los juicios que implican estimaciones y de los juicios en la aplicación de las políticas contables, los supuestos acerca del futuro, las causas de las incertidumbres en la estimación, así como su impracticabilidad, en cuanto corresponda, como resultado del estudio de casos no se identifica precisiones sobre los criterios en el juicio racional, aun cuando las estimaciones deban ser razonables. Desde la creencia racional, se requiere la afirmación de la creencia y su justificación. Asimismo, la clasificación de la probabilidad está limitada por lo general a tres niveles, se basa en el criterio profesional de la Gerencia y en la opinión de los asesores legales. Finalmente, desde la Teoría de Probabilidades, es posible aplicar sus definiciones y conceptos en la evaluación de contingencia, pues éstas involucran aleatoriedad, los desenlaces ocurridos en el pasado contribuyen a la información de la probabilidad previa y la probabilidad bayesiana mide la creencia sobre la ocurrencia de un evento, permitiendo la comparación de probabilidades obtenidas después de la ocurrencia de un evento de interés, siendo útil para la toma de decisiones. Esta investigación explora los conceptos matemáticos de la Teoría de Probabilidades proporcionando lineamientos para una creencia racional y su medición a nivel de la probabilidad bayesiana. La interpretación del concepto probable en las NIIF difiere del establecido en la Teoría de Probabilidades. Los rangos establecidos en la Guía de Implementación de la NIC 37 (probable, posible, remoto) no están acordes con la Teoría de Probabilidades, puesto que esta última se expresa entre los rangos 0 y 1 . La probabilidad de ocurrencia de un evento es necesaria en la evaluación de contingencias, pues estas involucran aleatoriedad, los desenlaces ocurridos en el pasado contribuyen a la información de la probabilidad previa y la probabilidad bayesiana provee una mayor base de entendimiento sobre los fenómenos de las ciencias sociales, por lo cual se constituye en una herramienta útil para la toma de decisiones; asimismo, debe considerar la naturaleza y características de la contingencia. Tanto la expresión matemática de la probabilidad como su interpretación adquieren importancia al momento de su análisis. La NIC 37 como el Marco Conceptual (2018) solo hacen referencia a una estimación razonable, dejando una brecha para la interpretación subjetiva de la probabilidad. Por ello, es necesario contar con información detallada a nivel de bases de datos histórica, de continua actualización y libre acceso, con el sumario de las contingencias, su tipificación, tiempo de resolución, tipo de estado en la instancia en la que se encuentra, resultados por sentencia firme, que permita la estimación de la probabilidad en términos matemáticos; así como, la rigurosidad en la revelación de información en las notas a los estados financieros según los requerimientos de las NIIF. La Gerencia y los especialistas deben considerar la legislación, la jurisprudencia, los resultados pasados, la información disponible, el juicio, experiencia y conocimiento del concepto de probabilidad bayesiana.

\section{REFERENCIAS}

Bernardo, J. y Smith, A. (2000). Bayesian Theory. England: John Wiley \& Sons, Ltd.

McGrayne, S. (2012). La teoría que nunca murió (T. Fernández y B. Equibar, trads.) Barcelona: CRÍTICA, S.L.

Compañía Minera San Ignacio de Morococha S.A.A. (2018). Estados financieros separados al 31 de diciembre de 2018 y 2017. Lima: Superintendencia de 
Mercado de Valores. http://www.smv.gob.pe/ConsultasP8/temp/Inf.\%20Financiera\%20Auditada\%20 Pwc\%202017.pdf

García, M. (2015). Introducción a la Teoría de la Probabilidad. Primer curso. México, D.F.: Fondo de Cultura Económica.

International Accounting Standard Board. (2020). Consejo Normativo de Contabilidad - NIIF. Consejo Normativo de Contabilidad: https://www.mef.gob.pe/contenidos/conta_publ/con_nor_co/nic/SpanishRed2020_ IAS37_GVT.pdf

International Accounting Standard Board. (2018). Consejo Normativo de Contabilidad - NIIF. Obtenido de Consejo Normativo de Contabilidad: https://www. mef.gob.pe/contenidos/conta_publ/con_nor_co/AnnotatedRB2019_A_ES_cf.pdf
International Accounting Standards Board. (2015). Normas Internacionales de Información Financiera. Parte A. Londres: IFRS Foundation.

Mosterín, J. (2008). Lo mejor posible. Racionalidad y Acción Humana (3ra ed.). Madrid: Alianza Editorial, S.A.

Sociedad Minera Corona S.A. (2018). Estados financieros al 31 de diciembre de 2018 y 2017. Lima: Superintendencia de Mercado de Valores. http://www.smv.gob. pe/ConsultasP8/temp/EEFF\%20Corona_Al\%203112-2017.PDF

Sociedad Minera El Brocal S.A.A. (2018). Estados financieros al 31 de diciembre de 2018 y 2017 junto con el dictamen de auditores independientes. Lima: Superintendencia de Mercado de Valores. http://www.smv.gob.pe/ConsultasP8/temp/El\%20Brocal\%20SAA\%2031-12-17-16\%20 IFRS\%20(espa\%C3\%B1ol).pdf 